\title{
Efficiency of translation termination in humans is highly dependent upon nucleotides in the neighbourhood of a (premature) termination codon
}

\author{
Frederic Pacho, ${ }^{1,2}$ Giovanna Zambruno, ${ }^{3}$ Valentina Calabresi, ${ }^{3}$ Dimitra Kiritsi, ${ }^{4}$ \\ Holm Schneider ${ }^{1}$
}

- Additional figures are published online only. To view these files please visit the journal online (http://jmg.bmj. com).

${ }^{1}$ Division of Molecular

Pediatrics, Children's Hospital, University of Erlangen-Nürnberg, Erlangen, Germany ${ }^{2}$ Institute of Molecular Biology, Center for Molecular Biosciences, University of Innsbruck, Austria

${ }^{3}$ Laboratory of Molecular and Cell Biology, Istituto

Dermopatico dell'Immacolata, Rome, Italy

${ }^{4}$ Department of Dermatology, University Medical Center Freiburg, Germany

\section{Correspondence to} Dr Holm Schneider, Children's Hospital, University of Erlangen-Nürnberg, Loschgestr 15, 91054 Erlangen, Germany: holm.schneider@uk-erlangen. de

Received 20 January 2011 Revised 17 May 2011 Accepted 26 May 2011 Published Online First 21 June 2011

\begin{abstract}
Background Spontaneous read-through of a premature termination codon (PTC) has so far not been observed in patients carrying nonsense mutations. This report describes a patient with junctional epidermolysis bullosa who was expected to die because of compound heterozygous nonsense mutations in the gene LAMA3 (R943X/R1159X), but was rescued by spontaneous readthrough of the R943X allele.

Results and conclusion FACS analysis of cells carrying various PTCs surrounded by their natural neighbouring codons revealed significant reporter gene expression despite the PTC only for this patient's genetic context. Gene expression could be abolished by replacing the first or third nucleotide before, or one of the two nucleotides following the PTC. Site-directed mutagenesis was used to identify genotypes allowing PTC read-through. The genetic context of the LAMA3 mutation R943X is close to a hypothetical consensus sequence for maximum PTC read-through. Bioinformatic analysis showed that this consensus sequence is present in four sequences from the NCBI reference database, each of which contains another in-frame termination codon three or four codons apart. This indicates strong selective pressure against leaky termination codons in the human genome. This patient's mutated full length mRNA escaped nonsensemediated decay, leading to LAMA3 mRNA levels similar to those of a healthy control, and full length laminin $\alpha 3$ could be detected in culture supernatant of the patient's keratinocytes. Immunofluorescence analyses of skin biopsies and continuous clinical improvement of the patient's condition suggested accumulation of intact laminin-332 in the epidermal basement membrane. These findings provide important clues for the prediction of PTC read-through in human genetic disease.
\end{abstract}

Homozygous or compound heterozygous nonsense mutations before the last exon of genes which code for structural proteins normally result in the absence of the gene product. The basis for this study was a patient with compound heterozygous nonsense mutations in the gene $L A M A 3$ encoding the $\alpha 3$ chain of laminin-332, who showed deposition of laminin-332 with full length $\alpha 3$ chains in the epidermal basement membrane, which had never been observed before.

Mutations in the laminin-332 genes, LAMA3, $L A M B 3$ and $L A M C 2$, are known to cause junctional epidermolysis bullosa (JEB). Individuals with mutations creating a premature termination codon (PTC) on both alleles of one of these genes usually display the lethal Herlitz type of JEB (HJEB; MIM 226700) and die in infancy due to widespread skin blistering, confluent erosions, loss of fluid, cells and proteins, and severe infections. ${ }^{1-3}$

Our patient showed the clinical phenotype of JEB with mechanically induced skin blistering, mucosal blisters, and dystrophic fingernails and toenails with paronychia (figure 1A) soon after birth. We detected known compound heterozygous PTC mutations, R943X ${ }^{4}$ and $\mathrm{R} 1159 \mathrm{X}^{5}$ in LAMA3 (figure 1B), suggesting the diagnosis of Herlitz JEB. Surprisingly, repeated immunofluorescence analyses of skin biopsies using antibodies against the individual chains of laminin-332 demonstrated the presence of this anchoring protein in the basement membrane of the patient's epidermis (figure 1C; staining of the first biopsy shown in supplementary figure 1). There were no clinical indications for revertant mosaicism. ${ }^{6}$ The patient survived infancy and his condition improved. He showed some catch-up growth and more and more normal development and is now able to attend a primary school (figure 1D).

After obtaining informed consent, keratinocyte cultures were established and RNA from these keratinocytes was investigated. Reverse transcriptase PCR (RT-PCR) amplification of $L A M A 3$ fragments containing one or both mutations produced specific bands (figure 1E), while no splice variants leading to internally shortened transcripts were observed which could have explained the milder phenotype. ${ }^{8}$ Cloning of the entire PCR product yielded a fragment with the expected size in 51 of 55 clones. To distinguish between the paternal R943X and the maternal R1159X allele, we used the restriction endonuclease Sal1, the natural recognition site of which is destroyed by the mutation R943X. None of the clones was cleaved by this enzyme, indicating that most mRNA molecules carried the mutation R943X.

Quantitative PCR analysis of LAMA3 transcription in the patient's keratinocytes revealed mRNA levels similar to those of a healthy control (figure 1F). To determine the ratio of R943X and R1159X alleles, PCR product was digested in parallel with Sal1 and with the restriction endonuclease BamH1, cleaving both alleles. Subsequent quantitative PCR amplification proved that the vast majority of specific mRNA was derived from 


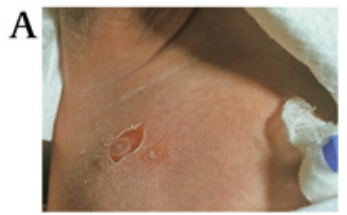

B

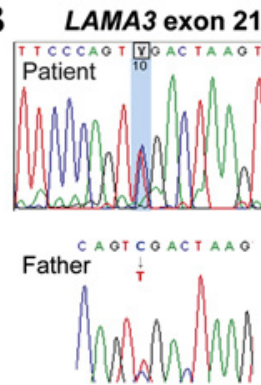

TGA $\rightarrow$ R943X

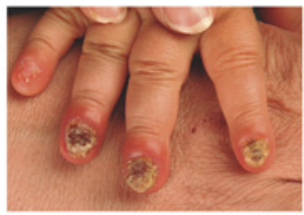

LAMA3 exon 26

CAOCTTOTOVOACTOCC
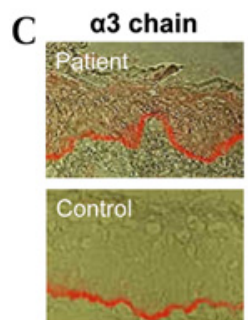
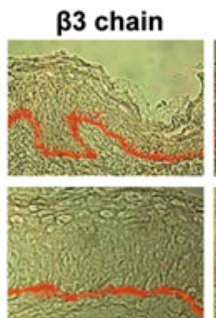

Mother

TTOTOCOATC,

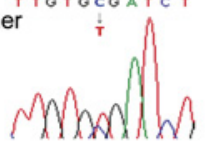

$\mathrm{TGA} \rightarrow \mathrm{R} 1159 \mathrm{X}$
D

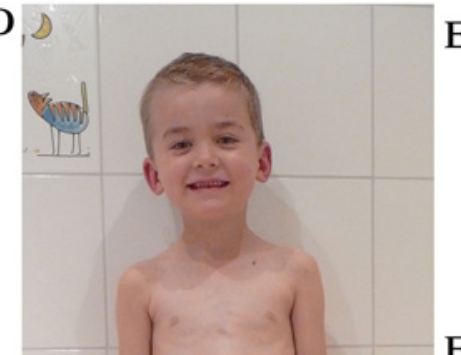

F

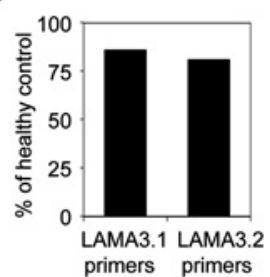

E

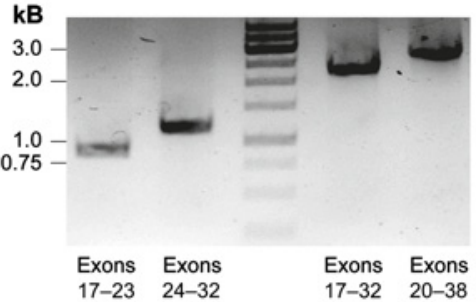

G

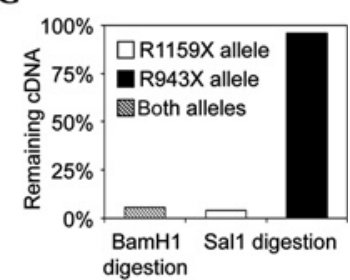

$\mathrm{H}$
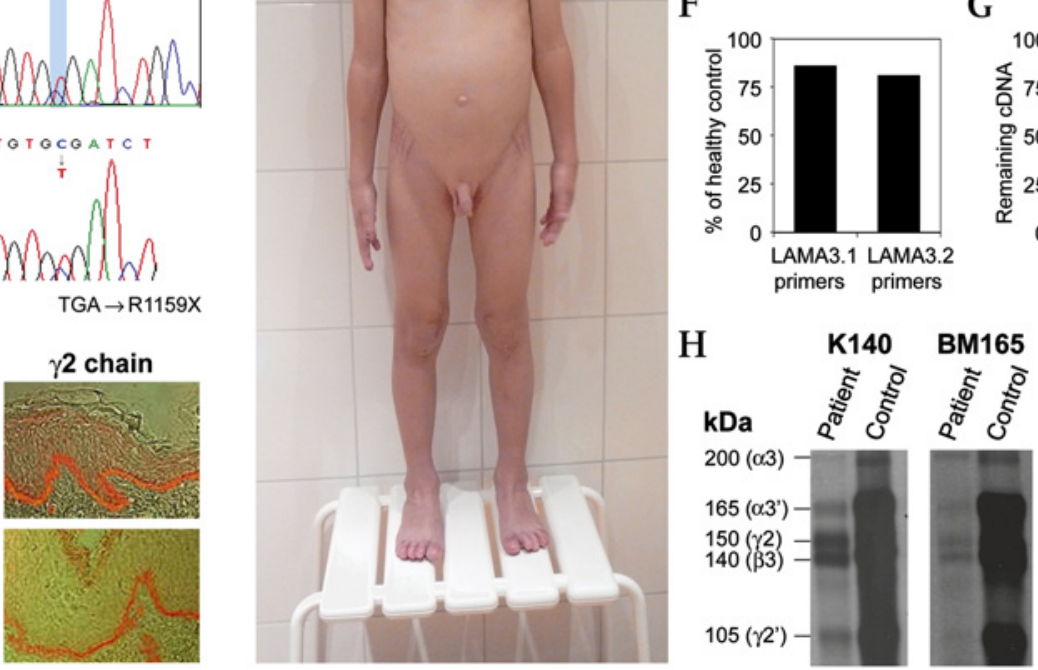

Figure 1 Genotype-phenotype discrepancy in a child with junctional epidermolysis bullosa. (A) Characteristic clinical picture in the first weeks of life: mechanically induced skin blistering and dystrophic finger nails with paronychia. (B) Electropherograms showing the patient's compound heterozygous mutations c.2827C $\rightarrow T(R 943 X)$ and c.3475C $\rightarrow T$ (R1159X) (upper panel) and the respective mutations in his clinically unaffected father and mother (lower panel). (C) Immunofluorescence analysis of a skin biopsy demonstrating the presence of the individual chains of laminin-332 (red) in the epidermal basement membrane of the patient at 5 years of age. Polyclonal antibody $\alpha 3$ llla recognising the $\mathrm{N}$-terminus of the $\alpha 3$ chain, monoclonal antibody 6F12 recognising the $\beta 3$ chain, and monoclonal antibody $\gamma 2 \mathrm{~m}$ were used to detect laminin $\alpha 3, \beta 3$ and $\gamma 2$, respectively. (D) Whole body aspect of the patient at school age showing no areas of active skin blistering. (E) PCR amplification of LAMA3 cDNA fragments from patient's keratinocytes resulted in distinct products of the expected size indicating insignificance of alternative splice products without the affected exons 22 or 27. (F) Quantitative PCR analysis of LAMA3 transcription in the patient's keratinocytes with different primer pairs showed that his mRNA levels were similar to those of a healthy control. (G) Removal of a Sal1 cleavage site by the mutation R943X was used to distinguish mRNA alleles by qPCR, proving that the LAMA3 mRNA was derived from the R943X allele. $(\mathrm{H})$ Immunoprecipitation assays with antibodies recognising the laminin chains $\beta 3$ (K140) or $\alpha 3$ (BM165) revealed the presence of full length ( $\alpha 3)$ and processed ( $\left.\alpha 3^{\prime}\right)$ laminin $\alpha 3$, in addition to laminin $\beta 3$ and $\gamma 2$ (full length, $\gamma 2$, and processed, $\left.\gamma 2^{\prime}\right)$ chains, in culture supernatant of radiolabelled keratinocytes from the patient (lanes 1 and 3 ) and a healthy control (lanes 2 and 4 ).

the R943X allele (figure 1G), indicating that mRNA with the mutation R1159X is efficiently cleared through nonsensemediated decay (NMD), while the R943X allele overcomes NMD to a large extent.

Translation of mRNA with the mutation R943X was expected to result in a truncated laminin $\alpha 3$ protein lacking approximately 40 amino acids of the laminin globular domain 1 and the entire globular domains 2 to 5 . However, in culture supernatant of the patient's keratinocytes only full length and regularly processed laminin $\alpha 3$ was detectable by immunoprecipitation with specific antibodies, although much less than in the supernatant of keratinocytes from a healthy control (figure $1 \mathrm{H}$ ). The continuous improvement of the patient's condition nevertheless indicated that translational PTC read-through allowed deposition and accumulation of a sufficient amount of fully functional laminin-332 molecules in the epidermal basement membrane, outweighing laminin-332 turnover. This conclusion was supported by immunofluorescence analyses of three skin biopsies, which demonstrated orthotopic, apparently increasing deposition of laminin-332 in the patient's epidermis, and by a previous report of a correlation between laminin-332 secretion and clinical severity. ${ }^{9}$ It has been in agreement with the clinical course, as the patient is now 6 years old and suffers only occa- sionally from localised skin blistering and erosions, mainly at high ambient temperatures.

To elucidate why full length laminin a3 protein could be produced, various PTCs surrounded by their natural neighbouring codons were cloned between an ATG codon and an enhanced green fluorescent protein (EGFP) reporter gene driven by a cytomegalovirus (CMV) promoter and introduced stably into HEK293 cells by retroviral gene transfer ${ }^{10}$ (supplementary figure 2). Fluorescence-activated cell sorting (FACS) analysis of the resulting cell lines revealed reporter gene expression only for the genetic context of our patient (figure 2A, lower left panel). The respective cells showed a significant fluorescence shift $(p<0.0001)$ in comparison to cells carrying a frameshift mutation in front of the EGFP gene. The native $L A M A 3$ sequence at this site served as positive control (figure 2A, lower right panel).

The phenomenon of spontaneous stop codon read-through has been described in bacteria and yeast, ${ }^{11}{ }^{12}$ and in eukaryotic cells for sequences derived from viruses, ${ }^{13} 14$ but has not been observed in patients carrying nonsense mutations. The efficacy of such read-through was shown to depend on the genetic context of the stop codon. Systematic investigation of read-through of the amber (TAG) termination codon indicated that the six nucleotides preceding and following the TAG determine read-through 

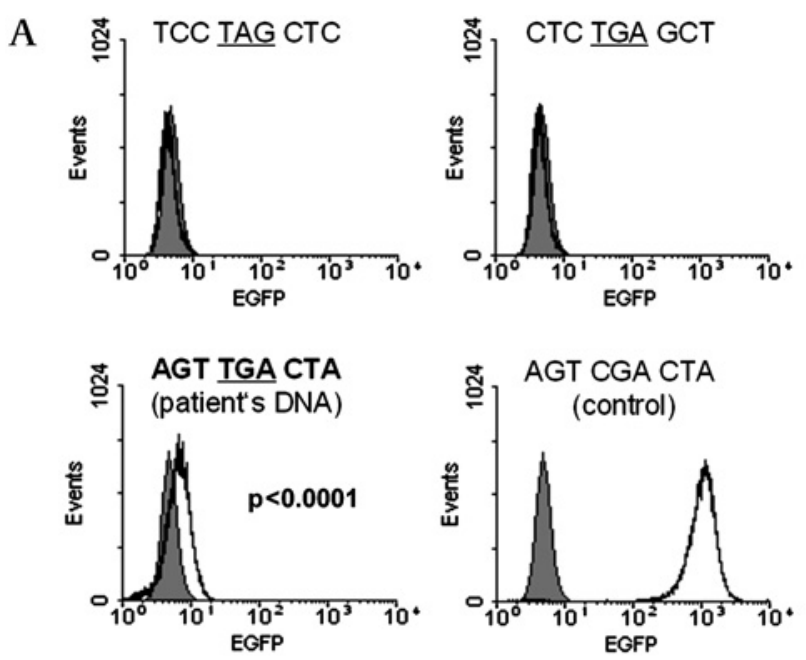

B

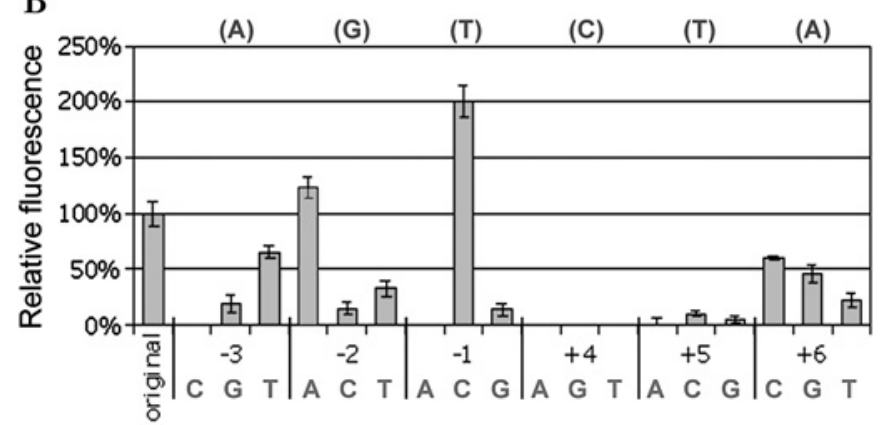

C

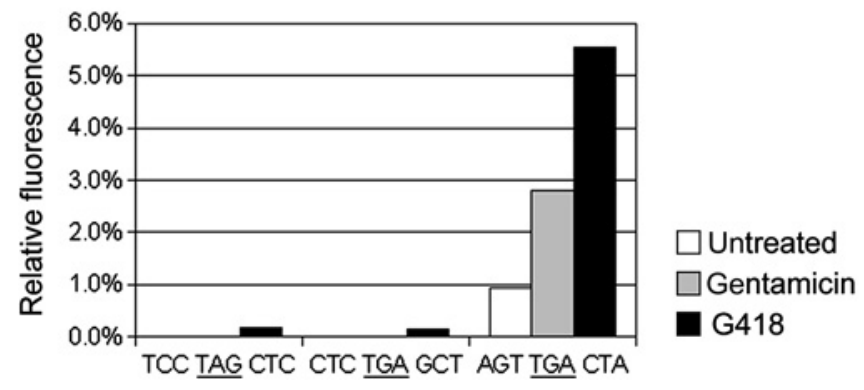

Figure 2 Influence of the genetic context of a stop codon on spontaneous read-through. (A) Flow cytometry histograms showing enhanced green fluorescent protein (EGFP) reporter gene expression as an indicator of read-through in various premature termination codon (PTC) mutations to depend on the nucleotides surrounding the termination codon (unfilled graphs) in comparison with negative controls (grey). (B) Relative levels of stop codon read-through after replacement of individual nucleotides from the patient's sequence (original). Reporter gene expression of the individual cell lines was normalised to ß-actin. Nucleotide substitutes and their position to the stop codon are indicated. (C) Context-dependent induction of read-through by the aminoglycosides gentamicin and G418 at concentrations of 4.0 or $0.4 \mathrm{mg} / \mathrm{ml}$, respectively (relative to the AGT CGA CTA control).

efficacy in yeast. ${ }^{15} 16$ The nucleotide immediately behind the stop codon appeared to be crucial, as there is striking evidence that the release factors (RF1/RF2 and eRF1 in prokaryotes and eukaryotes, respectively) also recognise the base at that position. ${ }^{17} 18$

To investigate which positions of the genetic context of a stop codon affect its spontaneous read-through in human cells, the three nucleotides upstream and downstream were replaced individually by each other nucleotide. Gene expression could be reduced to background levels by changing the first or third nucleotide before, or one of the two nucleotides following, the TGA (figure $2 \mathrm{~B}$ ). At position -3 , a cytosine was found to be most detrimental to read-through $(p<0.002)$, whereas at position -1 cytosine enhanced read-through $(p<0.005)$ in contrast to adenine or guanine. A cytosine immediately behind the stop codon appears to be a prerequisite of spontaneous read-through, as all other nucleotides abrogate it completely $(p<0.0005)$. At position +5 , both adenine and guanine reduced read-through to background levels. The third nucleotide behind the stop codon may vary. Most interestingly, our site-directed mutagenesis approach identified genetic contexts, AAT TGA CTA and AGC TGA CTA, which allow even more efficient read-through than the original sequence of the patient. These data demonstrate that in eukaryotic systems the impact of a TGA stop codon strongly depends on its genetic context. As aminoglycosides are known to induce PTC read-through, the effects of gentamicin and G418 on read-through of the TGA in our patient's context were evaluated. Read-through could be increased up to three- and six-fold, respectively (figure $2 \mathrm{C}$ ).

In our patient, a sufficient amount of $L A M A 3$ mRNA with the mutation R943X escapes from NMD, because the PTC is ignored and translation terminates at the normal stop codon, thereby removing signals that would otherwise assign the mRNA to $\mathrm{NMD}$. The rate of escape may depend on the efficacy of recognition of the mutated mRNA and its degradation by the NMD machinery in relation to the frequency of translation: the lower this ratio, the more likely escape occurs, because the probability of read-through increases with the number of translational events. Additionally, $L A M A 3$ transcription may be regulated by a negative feedback loop similarly to laminin $\beta 3,{ }^{19}$ which would prolong transcription in our patient and help to accumulate laminin-332 protein in the basement membrane. Once integrated into the basement membrane, laminin-332 is commonly thought to be relatively stable.

However, the LAMA3 mutation R943X had been described earlier by McLean et $a l^{4}$ in a patient with laryngo-onychocutaneous syndrome, who carried this mutation heterozygously with the mutation 151 insG, and we wondered why no evidence of spontaneous read-through of R934X had been found in this case. McLean et al showed that the mutation 151insG does not lead to nonsense mediated decay of the LAMA3 mRNA, but results in the production of an $\mathrm{N}$-terminally truncated laminin a3 protein. Considering our real-time PCR data and normal allelic transcription, $50 \%$ of the mRNA in the patient reported previously should be derived from the 151 insG allele, and $50 \%$ from the R943X allele. However, protein levels should be roughly $99.5 / 0.5 \%$, assuming $1 \%$ read-through. In our patient, more than $97 \%$ of the LAMA3 mRNA originated from the R943X allele, C-terminally truncated laminin $\alpha 3$ protein cannot be secreted, and significantly higher LAMA3 mRNA levels might be induced by the fact that laminin-332 is missing in the basement membrane. Taken together, the proportion of fully functional laminin-332 in our patient is close to $100 \%$. Most likely read-through was just missed by McLean et al, ${ }^{4}$ because $0.5 \%$ of fully functional laminin $\alpha 3 a$ may have had no clinically relevant impact on the LOC phenotype. This might be the reason why McLean et al reported only data on genomic DNA of their patient with the mutations 151insG and R943X.

To date a consensus sequence for efficient stop codon readthrough has only been determined for TAG in yeast. ${ }^{15}{ }^{16}$ It resembles the motif first described in tobacco mosaic virus, ACA CAA UAG CAA UUA. ${ }^{20}$ The consensus sequence for TGA readthrough deduced from our data, (A/T) (A/G)(T/C) TGA CTA, 
corresponds with the sequence used by Sindbis virus, TAC TGA CTA, ${ }^{13}$ which was shown to allow PTC read-through in human cells. For TAG surrounded by CAA we could verify low levels of read-through also in HEK293 cells (data not shown). In contrast, TGA in the same context inhibited read-through as expected from our consensus sequence.

The reason for the sequence specificities attributed to the different stop codons may lie in the specific tRNA(s) delivering amino acids in replacement for the TGA (Arg, Cys, Trp, Ser) or TAG codon (GIn). ${ }^{21} 22$ Under normal conditions the affinity of a near-cognate acyl-tRNA to the A-site of the ribosome is low. Its poor binding and efficient proofreading mechanisms of the ribosome normally prevent integration of wrong amino acids. However, nucleotides before and behind the PTC may interact with nucleotides of the tRNA anticodon loop, thereby stabilising codon-anticodon binding. This could prolong the contact time between tRNA and the A-site of the ribosome, facilitating the incorporation of an amino acid substitute. Interaction of a near-cognate anticodon with the TGA codon may be strengthened further by naturally occurring modifications of nucleotides forming the anticodon loop of many tRNAs, which lead to less restrictive codon recognition. ${ }^{23}$ Another possible explanation is the induction of conformational changes in the ribosome by nucleotides surrounding the PTC, mimicking the conformation when a cognate tRNA is bound. ${ }^{24}$

Bioinformatic analysis of the human refseq database revealed only 4/19885 mRNAs with a TGA termination codon embedded in the $A(A / G)(T / C)-C T A$ context as defined in this study. Surprisingly, every mRNA had a second in-frame TGA three or four triplicates later, which indicates a strong selective pressure against a genetic context allowing read-through.

This is the first report on the impact of each of three nucleotides upstream and downstream of an opal termination codon, suggesting a consensus sequence for significant read-through in human cells and in vivo. Our findings have important implications for patients with genetic diseases, as we provide the first evidence that spontaneous read-through may lead to much milder phenotypes than currently predicted for PTC mutations. Furthermore, our data may expedite the development of novel therapeutic attempts utilising the potential of aminoglycoside derivatives and non-aminoglycoside compounds to increase the frequency of such read-through. ${ }^{25}$

\section{METHODS}

\section{Skin biopsies}

The parents of our patient gave written informed consent to participate in this study. Punch biopsies were obtained from the skin of his left thigh at an age of 1, 2.5 and 6 years. Isolation of primary keratinocytes and immunoprecipitation were carried out as described previously. ${ }^{26}$

\section{Immunofluorescence studies}

Skin biopsies were embedded in OCT compound (Sakura Finetek) and sectioned on a Leica cryotome (Wetzlar, Germany). Cryosections were fixed for $5 \mathrm{~min}$ in ice-cold acetone. Afterwards the sections were blocked with $1 \%$ bovine serum albumin (BSA) in phosphate-buffered saline (PBS) and stained with the first antibody, diluted in PBS for $1-2 \mathrm{~h}$ at room temperature. Sections were then washed three times for 5 min with PBS, followed by incubation with the second antibody for $1 \mathrm{~h}$.

Following careful washing the sections were investigated under a fluorescence microscope (Zeiss, Jena, Germany).

\section{RNA analysis and quantitative PCR}

RNA was isolated with the EZNA Total RNA kit. cDNA was synthesised with Fermentas' First Strand cDNA Synthesis kit according to the manufacturer's instructions. Primers were designed to amplify the sequence encoding the $\alpha 3$ chain of laminin-332 (GenBank Accession number NM 198129.1). For quantitative PCR the FastSybr Master Mix (Applied Biosystems, Darmstadt, Germany) and the following primers were used: qPCR-LAMA3.1_forward ATGTTGGAGGTTACCCACCTG qPCR-LAMA3.1_reverse TCCTTCCTCCTTCTACAAGGCT qPCR-LAMA3.2_forward AGGTGCTCCAGCCAATTTGAC qPCR-LAMA3.2_reverse TAGGCTTGGGTTACTGGTCAGG qPCR-PPIA_forward CTTCACACGCCATAATGGCAC qPCR-PPIA_reverse TGCCATCCAACCACTCAGTC qPCR-TBP_forward AGCCAAGAGTGAAGAACAGTCC qPCR-TBP_reverse GAGCACAAGGCCTTCTAACCT qPCR-ACTB forward CTTCCTTCCTGGGCATGGAG qPCR-ACTB_reverse CTCCTTCTGCATCCTGTCGG qPCR-EGFP_forward AAGGTGAACTTCAAGATCCG qPCR-EGFP_reverse CTTCTTGTACAGCTCGTCCA

Quantitative PCR was performed on an iCycler (Bio-Rad, Munich, Germany). The samples were subjected to $40 \mathrm{PCR}$ cycles $\left(95^{\circ} \mathrm{C}\right.$ for $3 \mathrm{~min}, 95^{\circ} \mathrm{C}$ for $5 \mathrm{~s}, 60^{\circ} \mathrm{C}$ for $\left.30 \mathrm{~s}\right)$. For analysis of mRNA species of the patient, single-stranded cDNA was produced and converted to double-stranded, cleavable DNA by preamplification with the primers CCTCTTGGGATGGGAATTTG and TGATGGGTATCCATGGTCAG $\left(95^{\circ} \mathrm{C}\right.$ for $2 \mathrm{~min}, 95^{\circ} \mathrm{C}$ for $20 \mathrm{~s}, 55^{\circ} \mathrm{C}$ for $20 \mathrm{~s}, 72^{\circ} \mathrm{C}$ for $90 \mathrm{~s} ; 20$ cycles); $1 \mu \mathrm{l}$ of the PCR product was used for subsequent digestion with the restriction endonucleases Sal1 or BamH1, followed by quantitative PCR as described above. Undigested PCR product served as a control for normalisation.

\section{Generation of reporter cell lines}

DNA fragments consisting of a termination codon surrounded by its natural neighbouring codons or mutant sequences were cloned between an ATG codon and an EGFP reporter gene and introduced stably into 293 cells by retroviral gene transfer. Virus production and transduction into cells were carried out as described elsewhere. ${ }^{10}$ ExGen 500 was used for DNA transfection according to the manufacturer's protocol.

\section{Cell culture and flow cytometry}

Cells were maintained in Dulbecco's modified Eagle's medium (DMEM) with 10\% fetal calf serum (FCS) and 1\% penicillin/ streptomycin. For cell selection, puromycin was used at a final concentration of $1 \mu \mathrm{g} / \mathrm{ml}$. Before flow cytometry cells were trypsinised and washed carefully. They were resuspended in PBS and investigated on a FC500 cytometer (Beckman Coulter, Krefeld, Germany).

\section{Statistics}

All experiments were carried out in triplicate. To correct for different retroviral integration sites, EGFP mRNA levels were normalised to $\beta$-actin mRNA. Variability of mean fluorescence due to variations in laser intensity of the flow cytometer was accounted for by normalising to a positive control. Average SD of mean fluorescence intensity (mfi) was $5.35 \%$. Significance of $\mathrm{mfi}$ for different reporter constructs in comparison to $\mathrm{mfi}$ for the original sequence of the patient was determined by $t$ test. 


\section{Bioinformatic analysis}

The human refseq database was downloaded from ftp://ftp. ncbi.nih.gov/refseq/release/ and imported into a local mysql database. A short php-script was used to search for sequences with a TGA termination codon preceded by AGT, AGC, AAT or $\mathrm{AAC}$ and followed by CTA (script available upon request).

Acknowledgements We are grateful to the patient and his family for their cooperation, Elisabeth Koppmann for technical assistance, Manuel Koch (University of Cologne) for providing antibodies against laminin globular domain 2 and laminin 33, Takako Sasaki for antibody a3llla, Christiane Mühle, Michael Ausserlechner, Jung Park, Klaus von der Mark and Wolfgang Rascher for helpful suggestions, and Martin Zenker for critical reading of the manuscript.

Competing interests None.

Patient consent Obtained.

Contributors HS coordinated clinical data collection and follow-up examinations of the patient; GZ established cultures of the patient's keratinocytes; VC and DK performed immunoprecipitation assays and immunofluorescence analyses, respectively; FP planned and carried out experiments and analysed the data; HS supervised the experiments; FP and HS interpreted the data and wrote the manuscript.

Provenance and peer review Not commissioned; externally peer reviewed.

\section{REFERENCES}

1. Nakano A, Chao SC, Pulkkinen L, Murrell D, Bruckner-Tuderman L, Pfendner E, Uitto J. Laminin 5 mutations in junctional epidermolysis bullosa: molecular basis of Herlitz vs non-Herlitz phenotypes. Hum Genet 2002;110:41-51.

2. Mühle C, Jiang Q, Charlesworth A, Bruckner-Tuderman L, Meneguzzi G, Schneider $\mathrm{H}$. Novel and recurrent mutations in the laminin-5 genes causing lethal junctional epidermolysis bullosa: molecular basis and clinical course of Herlitz disease. Hum Genet 2005:116:33-42.

3. Schneider H, Mühle C, Pacho F. Biological function of laminin-5 and pathogenic impact of its deficiency. Eur J Cell Biol 2007;86:701-17.

4. McLean WH, Irvine AD, Hamill KJ, Whittock NV, Coleman-Campbell CM, Mellerio JE, Ashton GS, Dopping-Hepenstal PJ, Eady RA, Jamil T, Phillips RJ, Shabbir SG, Haroon TS, Khurshid K, Moore JE, Page B, Darling J, Atherton DJ, Van Steensel MA, Munro CS, Smith FJ, McGrath JA. An unusual N-terminal deletion of the laminin alpha3a isoform leads to the chronic granulation tissue disorder laryngo-onychocutaneous syndrome. Hum Mol Genet 2003:12:2395-409.

5. Varki R, Sadowski S, Pfendner E, Uitto J. Epidermolysis bullosa. I. Molecular genetics of the junctional and hemidesmosomal variants. J Med Genet 2006:43:641-52.

6. Pasmooij AMG, Pas HH, Bolling MC, Jonkman MF. Revertant mosaicism in junctional epidermolysis bullosa due to multiple correcting second-site mutations in LAMB3. J Clin Invest 2007;117:1240-8.

7. McGrath JA, Ashton GHS, Mellerio JE, Salas-Alanis JC, Swensson O, McMillan JR, Eady RA. Moderation of phenotypic severity in dystrophic and junctional forms of epidermolysis bullosa through in-frame skipping of exons containing non-sense or frameshift mutations. J Invest Dermatol 1999;113:314-21.

8. Gache Y, Allegra M, Bodemer C, Pisani-Spadafora A, de Prost Y, Ortonne JP, Meneguzzi G. Genetic bases of severe junctional epidermolysis bullosa presenting spontaneous amelioration with aging. Hum Mol Genet 2001;10:2453-61.

9. Matsui C, Pereira P, Wang CK, Nelson CF, Kutzkey T, Lanigan C, Woodley D, Morohashi M, Welsh EA, Hoeffler WK. Extent of laminin-5 assembly and secretion affect junctional epidermolysis bullosa phenotype. J Exp Med 1998; 187:1273-83

10. Pear WS, Nolan GP, Scott ML, Baltimore D. Production of high-titer helper-free retroviruses by transient transfection. Proc Natl Acad Sci USA 1993;90:8392-6.

11. Rospert S, Rakwalska M, Dubaquié Y. Polypeptide chain termination and stop codon readthrough on eukaryotic ribosomes. Rev Physiol Biochem Pharmacol 2005; 155:1-30.

12. Mottagui-Tabar $\mathbf{S}$, Isaksson LA. The influence of the $5^{\prime}$ codon context on translation termination in Bacillus subtilis and Escherichia coli is similar but different from Salmonella typhimurium. Gene 1998;212:189-96.

13. Li GP, Rice CM. Mutagenesis of the in-frame opal termination codon preceding nsP4 of Sindbis virus: studies of translational readthrough and its effect on virus replication. J Virol 1989;63:1326-37.

14. Cassan M, Rousset JP. UAG readthrough in mammalian cells: effect of upstream and downstream stop codon contexts reveal different signals. BMC Mol Biol 2001:2:3.

15. Namy 0, Hatin I, Rousset JP. Impact of the six nucleotides downstream of the stop codon on translation termination. EMBO Rep 2001;2:787-93.

16. Tork S, Hatin I, Rousset J, Fabret C. The major $5^{\prime}$ determinant in stop codon readthrough involves two adjacent adenines. Nucleic Acids Res 2004;32:415-21.

17. Poole ES, Major LL, Mannering SA, Tate WP. Translational termination in Escherichia coli: three bases following the stop codon crosslink to release factor 2 and affect the decoding efficiency of UGA-containing signals. Nucleic Acids Res 1998;26:954-60.

18. Howard MT, Shirts BH, Petros LM, Flanigan KM, Gesteland RF, Atkins JF. Sequence specificity of aminoglycoside-induced stop condon readthrough: potential implications for treatment of Duchenne muscular dystrophy. Ann Neurol 2000;48:164-9.

19. Marionnet C, Pierrard C, Vioux-Chagnoleau C, Sok J, Asselineau D, Bernerd F. Interactions between fibroblasts and keratinocytes in morphogenesis of dermal epidermal junction in a model of reconstructed skin. J Invest Dermato/ 2006;126:971-9.

20. Goelet P, Lomonossoff GP, Butler PJ, Akam ME, Gait MJ, Karn J. Nucleotide sequence of tobacco mosaic virus RNA. Proc Natl Acad Sci USA 1982;79:5818-22.

21. Feng YX, Copeland TD, Oroszlan S, Rein A, Levin JG. Identification of amino acids inserted during suppression of UAA and UGA termination codons at the gag-pol junction of Moloney murine leukemia virus. Proc Natl Acad Sci USA 1990;87:8860-3.

22. Chittum HS, Lane WS, Carlson BA, Roller PP, Lung FD, Lee BJ, Hatfield DL. Rabbit beta-globin is extended beyond its UGA stop codon by multiple suppressions and translational reading gaps. Biochemistry 1998;37:10866-70

23. Agris PF, Vendeix FAP, Graham WD. tRNA's wobble decoding of the genome: 40 years of modification. $J$ Mol Biol 2007;366:1-13.

24. Steitz TA. A structural understanding of the dynamic ribosome machine. Nat Rev Mol Cell Biol 2008;9:242-53.

25. Du L, Damoiseaux R, Nahas S, Gao K, Hu H, Pollard JM, Goldstine J, Jung ME, Henning SM, Bertoni $C$, et al. Nonaminoglycoside compounds induce readthrough of nonsense mutations. J Exp Med 2009;206:2285-97.

26. Castiglia D, Posteraro P, Spirito F, Pinola M, Angelo C, Puddu P, Meneguzzi G, Zambruno G. Novel mutations in the LAMC2 gene in non-Herlitz junctional epidermolysis bullosa: effects on laminin- 5 assembly, secretion, and deposition. $J$ Invest Dermatol 2001;117:731-9. 


\section{Efficiency of translation termination in humans is highly dependent upon nucleotides in the neighbourhood of a (premature) termination codon}

Frederic Pacho, Giovanna Zambruno, Valentina Calabresi, Dimitra Kiritsi and Holm Schneider

J Med Genet 2011 48: 640-644 originally published online June 21, 2011 doi: 10.1136/jmg.2011.089615

Updated information and services can be found at:

http://jmg.bmj.com/content/48/9/640

These include:

Supplementary Supplementary material can be found at:

Material http://jmg.bmj.com/content/suppl/2011/06/01/jmg.2011.089615.DC1.h tml

References This article cites 26 articles, 12 of which you can access for free at: http://jmg.bmj.com/content/48/9/640\#BIBL

Email alerting Receive free email alerts when new articles cite this article. Sign up in the service box at the top right corner of the online article.

\section{Collections}

Articles on similar topics can be found in the following collections

Clinical diagnostic tests (353)

Dermatology (240)

Genetic screening / counselling (879)

Immunology (including allergy) (601)

Molecular genetics (1249)

Surgery (105)

Surgical diagnostic tests (105)

Clinical genetics (255)

\section{Notes}

To request permissions go to:

http://group.bmj.com/group/rights-licensing/permissions

To order reprints go to:

http://journals.bmj.com/cgi/reprintform

To subscribe to BMJ go to:

http://group.bmj.com/subscribe/ 